\title{
DETECTING MALICIOUS DATA INJ ECTION IN EVENT DETECTION USING EADA SCHEME
}

\author{
M.Usha, P.Prittopaul ${ }^{1}$, R.Vijayalakshmi ${ }^{2}$ and T.Shanmuga Priya ${ }^{3}$
}

\begin{abstract}
Wireless Sensor system is made out of a few remote sensors, each of which screens particular ecological properties, records detecting information, determines natural conditions by amassing the detecting information, and returns the accumulated information to the base station. We propose a dynamic bunch head choice strategy, EADA which chooses the hubs having the limit an incentive over the normal.Cluster Head can be selected based on maximum available energy, minimum distance and having maximum throughput for preventing malicious nodes joining wireless sensor networks (WSNs), an access control mechanism is necessary for the trustworthy cooperation between the nodes. In addition to access control, recently, privacy has been an important topic regarding how to achieve privacy without disclosing the real identity of communicating entities in the WSNs. The proposed strategy is dynamic in nature as choice process is revived occasionally, which can ensure effective assault location and keep up agreeable system lifetime. In particular, we abuse the area data of sensors and arbitrarily select witnesses situated in a ring territory to confirm the authenticity of sensors and to report identified assaults.
\end{abstract}

Key words: EADA, Malicious nodes, trustworthy, Cluster Head

\section{INTRODUCTION}

A Wireless Sensor System is a gathering of sensor hubs to screen the physical or ecological surroundings, for example, Weight, sound, temperature and so forth... and send the detected information to the base station. The development of remote sensor systems was disturbed by military applications, for example, war zone reconnaissance; today such systems are utilized as a part of numerous mechanical and purchaser applications, for example, modern process observing and control, machine wellbeing checking, et cetera. The cost of sensor hubs is comparatively conflicting, running from a couple to many dollars, contingent upon the entanglement of the individual sensor hubs.. The topology of the WSNs can shift from a basic star system to a progressed multi-bounce remote work arrange. The proliferation strategy between the bounces of the system can defeat or flooding. As sensor hubs for occasion observing are unsurprising to work for quite a while without energizing their batteries, rest booking plan is constantly utilized amid the checking procedure. Discernibly, correspondence delay brought on by rest planning instrument in light of the fact that the sender hubs ought to hold up until the recipient hubs are dynamic and prepared to get the message.

The delay could be essential as the system estimate expands. The majority of rest booking technique concentrate on limiting the vitality utilization. In actuality, in the basic occasion checking, just few bundles should be transmitted amid more often than not. At the point when a huge occasion is identified, the caution bundle ought to be screen to the whole system as quickly as time permits. Along these lines, broadcasting delay is an essential issue for the use of the basic occasion checking. To limit the telecom delay, it is required to limit the time tired for holding up amid the spread. The perfect situation is the goal hubs wake up promptly when the source hubs get the telecom parcels.

\footnotetext{
${ }^{1}$ Department of CSE, Velammal Engineering College, India

${ }^{2}$ Department of CSE, Velammal Engineering College, India

${ }^{3}$ Department of CSE, Velammal Engineering College, India
} 


\section{LITERATURE SURVEY}

2.1 Detecting compromised nodes in wireless sensor networks

AUTHOR: M. Mathews, M. Song, S. Shetty, and R. McKenzie $\quad$.In this paper, an irregularity based interruption recognition framework to identify bargained hubs in remote sensor networks.

2.2. TITLE: Insider attacker detection in wireless sensor networks. AUTHOR: F. Liu, X. Cheng, and D. Chen The proposed calculation considers numerous properties at the same time in hub conduct assessment, with no necessity on an earlier learning about ordinary/malicious sensor exercises. In addition, it is application-accommodating, which utilizes unique estimations from sensors and can be utilized to screen numerous parts of sensor systems administration practices. Our calculation is simply limited, fitting admirably to the substantial scale sensor systems.

2.3. TITLE: A trust based framework for secure data aggregation in wireless sensor network.

AUTHOR: W. Zhang, S. K. Das, and Y. Yonghe,The trustworthiness (notoriety) of every individual sensor hub is assessed by utilizing a data theoretic idea, Kullback-Leibler (KL) separation, to recognize the traded off hubs through an unsupervised learning calculation. After amassing, a supposition, a metric of the level of conviction, is created to speak to the vulnerability in the total outcome. As the outcome is being spread and amassed through the courses to the sink, this sentiment will be engendered and controlled by Josang's conviction display. Taking after this model, the instability inside the information and accumulation results can be adequately evaluated all through the system. Reproduction comes about show that our trust based structure gives an effective instrument to distinguishing traded off hubs and thinking about the instability in the system.

2.4. TITLE: Energy-efficient surveillance system using wireless sensor networks

AUTHOR:Tian He, Sudha Krishnamurthy, John A. Stankovic

The concentration of observation missions is to procure and confirm data about adversary capacities and places of threatening targets. Such missions frequently include a high component of hazard for human work force and require a high level of stealthiness. Thus, the capacity to send unmanned reconnaissance missions, by utilizing remote sensor systems, is of incredible down to earth significance for the military. As a result of the vitality requirements of sensor gadgets, such frameworks require a vitality mindful plan to guarantee the life span of observation missions. Arrangements proposed as of late for this kind of framework show promising outcomes through recreations

\subsection{TITLE: System architecture of a wireless body area sensor network for ubiquitous health monitoring}

AUTHOR: C. Otto, A. Milenkovi'c, C. Sanders, and E. Joranov.Concentrate on anticipation and early recognition of ailment or ideal support of ceaseless conditions guarantee to enlarge existing social insurance frameworks that are generally organized and enhanced for responding to emergency and overseeing sickness as opposed to wellbeing. The expected change and developing new administrations are all around coordinated to help adapt to the impending emergency in the medicinal services frameworks brought on by current financial, social, and statistic patterns.

\subsection{TITLE: Deploying a wireless sensor network on an active volcano}

AUTHOR: G.Werner-Allen

Expanding overwhelming and control hungry information accumulation gear with help littler remote sensor organize hubs prompts quicker, bigger organizations. Exhibits containing many remote sensor hubs are currently conceivable, permitting logical reviews that aren't achievable with customary instrumentation. Outlining sensor systems to bolster volcanic reviews requires tending to the high information rates and high information devotion these reviews request. The creators' sensor-arrange application for volcanic information accumulation depends on activated occasion location and solid information recovery to meet transfer speed and information quality requests.

\subsection{TITLE: Secure routing in wireless sensor networks: Attacks and Countermeasures}

AUTHOR: C. Karlof and D. Wagner

Current proposition for directing conventions in sensor systems streamline for the restricted capacities of the hubs and the application particular nature of the systems, however don't consider security. In spite of the fact that these conventions have not been planned with security as an objective, we feel it is critical to investigate their security properties. At the point when the guard has the liabilities of shaky remote correspondence, constrained hub 
capacities, and conceivable insider dangers, and the foes can utilize capable tablets with high vitality and long range correspondence to assault the system, outlining a safe directing convention is non-inconsequential.

\subsection{TITLE:Attribute-Aware Data Aggregation Using Potential-BasedDynamic Routing in Wireless Sensor network}

AUTHOR:FengyuanRen,Jiao Zhang, Yongwei Wu

Wireless sensor networks (WSNs) can be promptly conveyed in different conditions to gather data in a selfgoverning way, and in this manner can bolster inexhaustible applications, for example, living space checking , moving target following, and fire discovery. WSNs are for the most part occasion based frameworks, and comprise of at least one sinks which is in charge of social affair particular information by sending questions. For the most part, sensor hubs are thickly conveyed and in charge of recognizing intriguing occasions and sending related information to sinks. The cooperative flag handling calculations can be composed in WSN applications to enhance the detecting execution.

\subsection{TITLE: Forest Fire Modeling and Early Detection Using Wireless Sensor Networks} AUTHOR: M. Hefeeda, M. Bagheri

Woods Climate Record (FWI) Framework, and show how its diverse segments can be utilized as a part of planning productive fire recognition frameworks. The FWI Framework is a standout amongst the most extensive woods fire risk rating frameworks in North America, and it is sponsored by quite a few years of ranger service explore. The examination of the FWI Framework could be of enthusiasm for its own particular ideal to analysts working in the sensor arrange region and to sensor makers who can enhance the correspondence and detecting modules of their items to better fit timberland fire location frameworks.

\subsection{TITLE: Monitoring Volcanic Eruptions with a Wireless Sensor Network}

AUTHOR: G. Werner-Allen

The system gathered infrasonic (low-recurrence acoustic) signals at $102 \mathrm{~Hz}$, transmitting information over a $9 \mathrm{~km}$ remote connection to a remote base station. Amid the organization, we gathered more than 54 hours of ceaseless information which included no less than 9 huge blasts. Hubs were time-synchronized utilizing a different GPS recipient, and our information was later connected with that gained at an adjacent wired sensor exhibit. Notwithstanding persistent testing, we have built up an appropriated occasion indicator that consequently triggers information transmission when an all around associated flag is gotten by various hubs.

\section{PROPOSED SYSTEM}

In existing framework, Wireless sensor networks (WSNs) are defenseless and can be vindictively bargained, either physically or remotely, with possibly pulverizing impacts. At the point when sensor networks are utilized to identify the event of occasions, for example, fires, interlopers, or heart assaults, pernicious information can be infused to make fake occasions, and consequently trigger an undesired reaction, or to veil the event of real occasions. A calculation is intended to discover malignant data infusions and estimation assesses that are impervious to a few sensors notwithstanding when they crash in the assault. They additionally proposed an approach to apply this calculation in various application settings and assess its outcomes on three diverse datasets drawn from unmistakable WSN organizations. This prompts recognize diverse tradeoffs in the outline of such calculations and how they are affected by the application setting.

\subsection{DISADVANTAGE}

- In existing strategies, increment in delay, hub disappointment, high information repetition and substantial measure of vitality usage emerges, since; it is utilizing flooding, tattling, coordinate correspondence.

- $\quad$ Detection malicious less exactness

To overcome this drawback, of edge an incentive in this proposed calculation. From the chose hubs, the hub with most extreme accessible vitality, at any rate separate and having greatest throughput is chosen as the bunch head. For preventing malicious nodes joining wireless sensor networks (WSNs), an access control mechanism is necessary for the trustworthy cooperation between the nodes. In addition to access control, recently, privacy has been an important topic regarding how to achieve privacy without disclosing the real identity of communicating entities in 
the WSNs. The proposed strategy is dynamic in nature as choice process is invigorated intermittently, which can ensure effective assault identification and keep up acceptable system lifetime. In particular, we abuse the area data of sensors and arbitrarily select witnesses situated in a ring zone to check the authenticity of sensors and to report distinguished assaults

\subsection{ADVANTAGES:}

- $\quad$ Improved security upgrades.

- $\quad$ Enhanced Assault discovery and counteractive action procedures.

- Throughput and parcel conveyance proportion (PDR) can be upgraded fundamentally.

- $\quad$ Reduced normal end-to-end defer and Steering overhead of messages.

\subsection{ALGORITHM STEPS}

\section{Step1:}

Input: Sensor 1...Sensor Output: Attackers Detection Based Wireless Sensor Network

\section{Step2:}

Sensors: , $\mathrm{C}_{\mathrm{M}}, \mathrm{CH} 1 \ldots \mathrm{N}$

Pest : Attacker1..N

Sink-Base Station

Step3:

BS Sensed to All Neighbors Discovered update every few seconds

(Location Information,Sensor information)

Cluster Member communicated to Base Station..Bs Collected Data From CH

Step4:

Apply EADA

Secure Transmission Path Transmission Proocess

Step5:

Some Nodes (Attackers) enter WSN Networks

Step 6:

Check Energy Level

If (Attackers $\leftarrow$ True)

Attackers spread infection near nodes

Blackhole, Wormhole Attackers

BS Destroyed to Attackers

Else

Normal Data Collection from Sensors

End if

\section{SYSTEM ARCHITECTURE}

Sensor nodes transmit the data to base station. Neighbor discovery is used to activate all the nodes in the network.

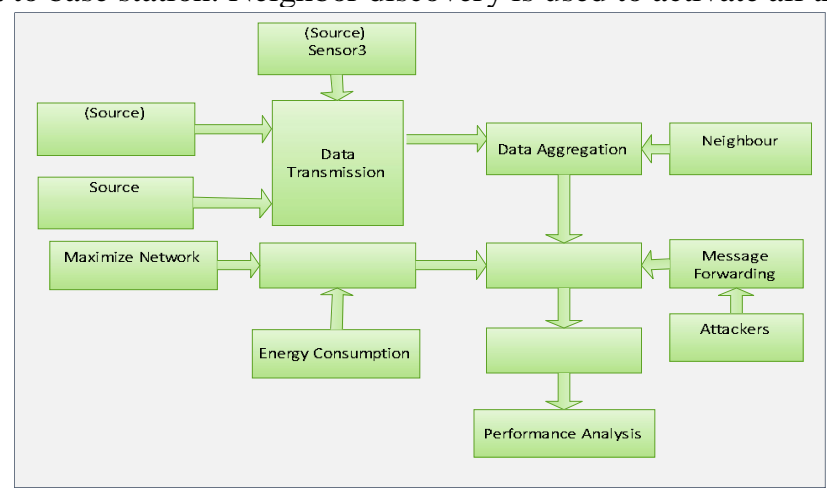

Fig.1. Architecture of proposed system 


\section{SYSTEM IMPLEMENTATION}

\subsection{Sensor Network formation}

This module manages formation of the $\mathrm{N}$ number of hubs that is mostly utilized as a part of this venture. The nodes are created dynamically according based on the query required by destination nodes. Every hub has unmistakable ID and the hub sort. Amid the formation of a hub everything about their neighbor hubs are put away. Current capacities of given remote sensor arrange, outline an information accumulation organize that would meet the logical prerequisites. Before sending the system each hub gathers the sensor hub subtle elements. In light of which the system is being shaped.

\subsection{Data Communication}

This Module is created to WSN systems information correspondence and accumulation handle. The radio and IEEE 802.11 Macintosh layer models were utilized. The system based information handling or most costly and information correspondence level on their execution on the system. Different sources are making sending bundles; every information has enduring size of 512 bytes. Every Sensor hub to move arbitrarily on their system, it's increasingly and most expectable on their systems.

\subsection{EADA}

This plan utilizes the idea of parcel characteristics which is utilized as an identifier of the information bundles created from different sensor hubs. Characteristic Id is relegated for every information parcels by this plan. Characteristic ID information parcels of same documents are gathered together. Actually, the least difficult approach to total information spilling out of the sources to the sink is to choose some uncommon hubs that work as conglomeration focuses and characterize a favored heading to be taken after when sending information. In this approach, a tree structure is built first to either course information gathered or reacts to inquiries produced by the sink. The collection is performed amid the routing, when at least two information bundles touch base at a similar hub of the tree. This hub gathers and totals the information then forward just a single bundle with the amassed data.

\subsection{Packet Driven Timing Algorithm}

To adjust to our dynamic steering convention and beat the downsides in existing timing schemes, we propose a packet-driven adaptive timing scheme. The hub has an inbuilt clock for the packets with same property in its line. At the point when the clock fires, the relating total is performed. At the point when a hub gets another packet the estimation of clock is instated or refreshed powerfully. This algorithm to stay away from unreasonable packet dropping. The timing control algorithm for our EADA is packet driven and adaptive.

\subsection{Dynamic Routing}

Route was found by utilizing attribute aware aggregation algorithm. To begin with source hub was chosen. For that hub set of neighbors found. From that neighbor, next sending hub is the hub that has same attribute as present sending hub. This procedure is preceded until achieving the sensor hub. At last information was exchanged through that way.

\section{IMPLEMENTATION RESULTS}

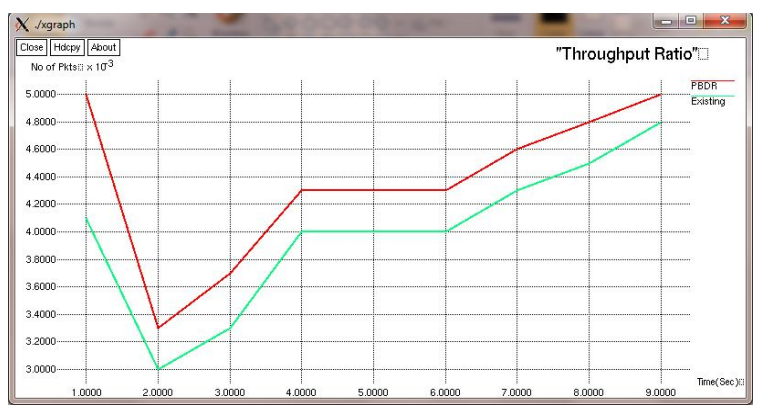


This graph shows the comparison of throughput between existing and proposed system.X-axis and $\mathrm{Y}$-axis represent time(in seconds) and number of packets.Initially,at time $2 \mathrm{sec}$ number of packets increases from 3000 to 3300.Between the time 4 to 6 seconds ,the packets are transferred as stable.Finally, it increases up to 20 percentage.

\section{PACKET DELIVERY RATIO}

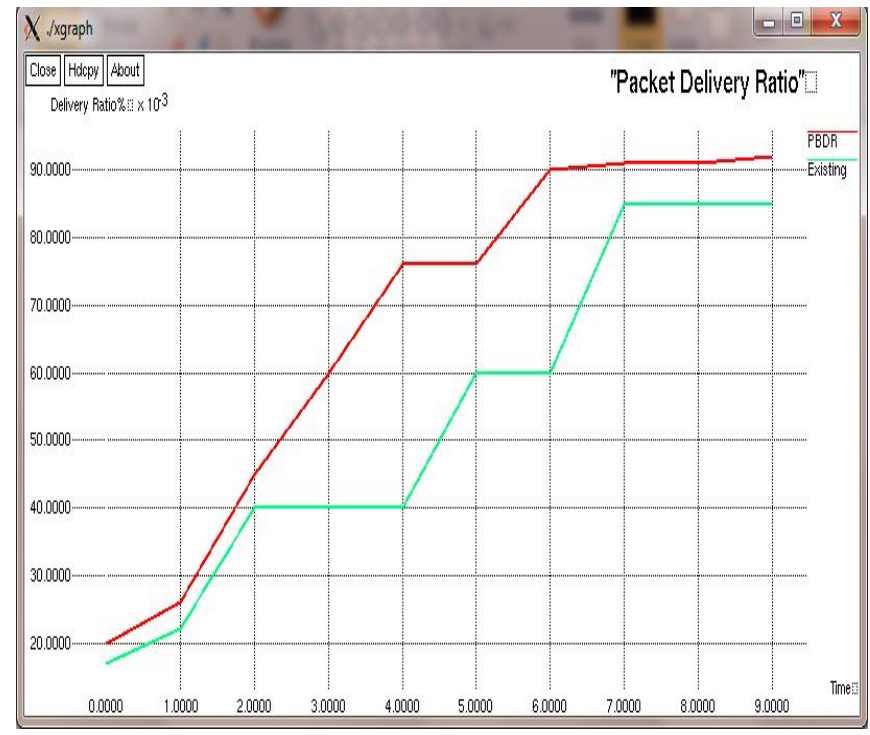

This graph shows the comparison of packet delivery ratio between existing and proposed system.X-axis and Y-axis represents time (in seconds) and number of packets delivered.The delivery ratio grows up exponentially from the time 1 to $4 \mathrm{sec}$.Atlast, the packets is delivered at stable.

AVERAGE END TO END DELAY

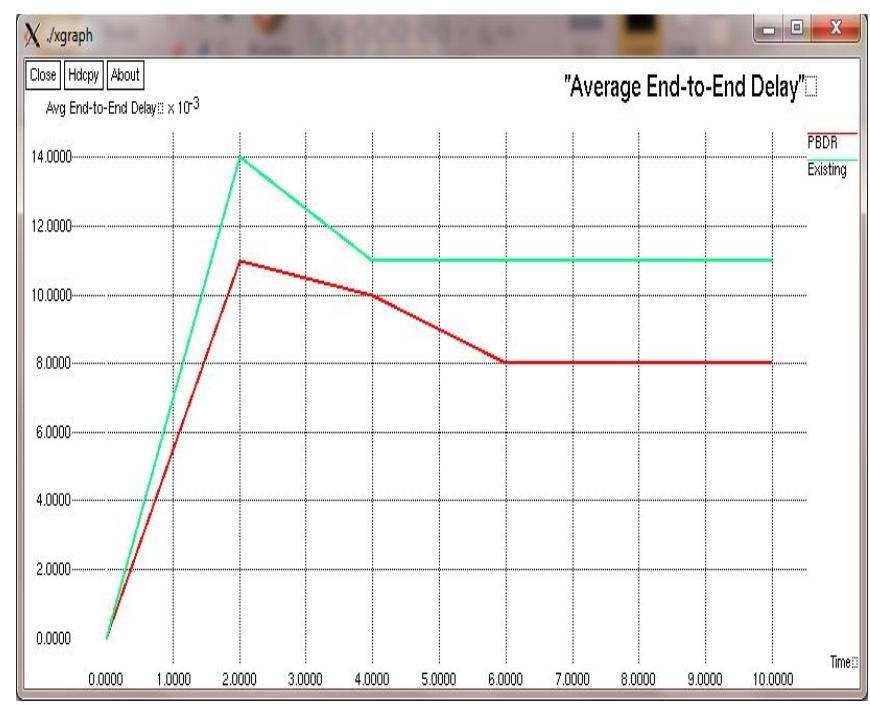

This graph shows the average end to end delay between existing and proposed system.X-axis and $\mathrm{Y}$-axis represents time(in seconds) and number of packets from time $6 \mathrm{sec}$ to $10 \mathrm{sec}$ the packet delay decreases. 


\section{ENERGY LEVEL}

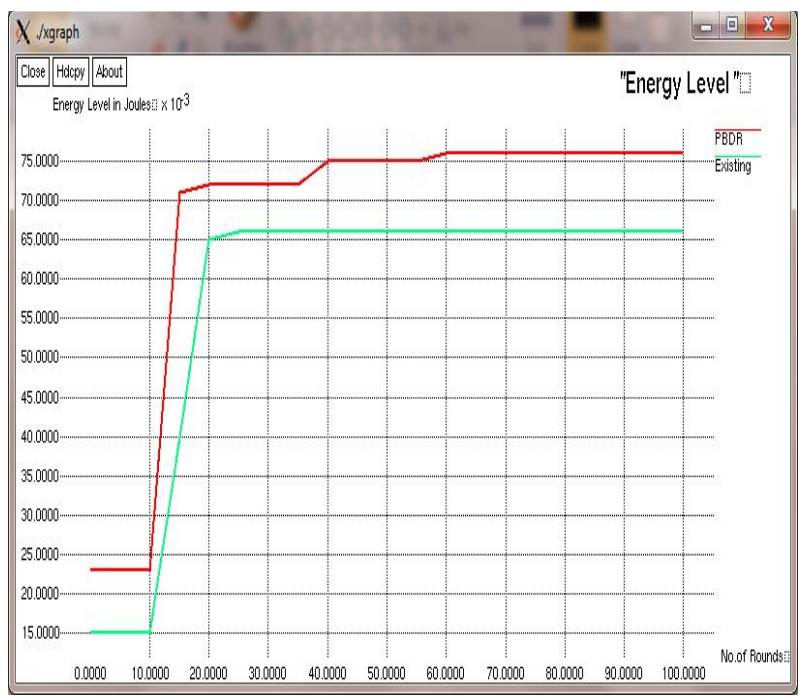

This graph shows the comparison of Energy level between existing and proposed system.X-axis and Y-axis represents number of rounds and energy level in joules.From round 10 the energy level between existing and proposed has been increased exponentially.

\section{CONCLUSION}

In this paper,the EADA scheme utilize the idea of parcel attributes which is utilized as an identifier of the information bundles produced from different sensor hubs.In this scheme attribute Id is assigned for each data packets. By this attribute ID information parcels of same records were gathered together. A cluster node is selected based on the maximum energy level and data are send from the sub nodes to cluster node which in turn send to base station. Base station will sense the nodes since it has information about the all the nodes. If attacker injects malicious data on a node that is in inactive state.Base station detects and eliminates the attacker node based on trustvalue.An adaptive packet driven timing control algorithm is proposed to give more opportunities to information total on hubs.

\section{REFERENCES}

[1]. W. Zhang, S. K. Das, and Y. Yonghe, "A trust based framework for secure data aggregation in wireless sensor networks," in Proc. 3rd Annu. IEEE SECON, 2006, pp. 60-69.

[2]. F. Liu, X. Cheng, and D. Chen, "Insider attacker detection in wireless sensor networks," in Proc. 26th IEEE INFOCOM, 2007, pp. 19731945.

[3]. M. Mathews, M. Song, S. Shetty, and R. McKenzie, "Detecting compromised nodes in wireless sensor networks," in Proc. SNPD, 2007, vol. 1, pp. 273-278.

[4]. T. He et al., "Energy-efficient surveillance system using wireless sensor networks," in Proc. MobiSys, 2004, pp. $270-283$.

[5]. C. Otto, A. Milenkovi'c, C. Sanders, and E. Joranov, "System architecture of a wireless body area sensor network for ubiquitous health monitoring," J. Mobile Multimedia, vol. 1, no. 4, pp. 307-326, Jan. 2005.

[6]. G.Werner-Allen et al., "Deploying a wireless sensor network on an active volcano," Internet Comput., vol. 10, no. 2, pp. 18-25, Mar./Apr. 2006.

[7]. C. Karlof and D. Wagner, "Secure routing in wireless sensor networks: Attacks and countermeasures," AdHocNetw., vol. 1, no. 2/3, pp. 293-315, 2003.

[8]. Perrig, J. Stankovic, and D. Wagner, "Security in wireless sensor networks," Mag. Commun., vol. 47, no. 6, pp. 53-57, Jun. 2004.

[9]. M. Hefeeda, M. Bagheri, "Forest Fire Modeling and Early Detection Using Wireless Sensor Networks", Ad Hoc and Sensor Wireless Networks, pp. 169-224, Apr. 2009.

[10]. G. Werner-Allen, "Monitoring Volcanic Eruptions with a Wireless Sensor Network", Proc. 2nd European Workshop Wireless Sensor Networks (EWSN 05), 2005. 